\title{
Characteristics of critically ill elderly patients admitted to a tertiary intensive care unit in Nigeria and outcome of management
}

\author{
Tobi KU*, Ndokwu EO and Edomwonyi NP* \\ Department of Anaesthesiology, University of Benin Teaching Hospital, Benin City, Nigeria
}

\begin{abstract}
Introduction: The management and outcome of elderly patients aged 65 years and above admitted to the intensive care unit (ICU) are often complicated by the presence of co-morbidities and reduced physiological reserve.

Methodology: This was a retrospective, case-control study. Patients aged 65 years and above admitted to the unit from January 2012 to June 2013 were included in the study. Admission and discharge register in the ICU was examined. A patient before and after each elderly patient were recruited to serve as controls in the study.

Result: Seventy-nine (79) elderly patients were admitted to the ICU and it constituted $12.6 \%$ of total ICU admission with a mortality rate of $49.6 \%$. Male: female ratio was 2:1. Postoperative care constituted the highest indication for ICU admission (41.8\%) followed by cerebrovascular accident (stroke), 12.6\%. Younger patients were about twice more likely to be mechanically ventilated than elderly patients. $(\mathrm{p}=0.05, \mathrm{OR}=1.855)$
\end{abstract}

Conclusion: The mortality rate of elderly patients admitted to the ICU was high. Appropriate admission criteria and protocol for the management of elderly patients in the ICU should, therefore, be developed to improve outcome. $Z$

\section{Introduction}

The management and outcome of elderly patients aged 65 years and above who are admitted to the intensive care unit (ICU) are often complicated by the presence of co-morbidities and reduced physiological reserve. It has been shown that severe sepsis which often requires ICU care increases with age [1]. In addition, the risk of hospital admission due to pneumonia has been found to double in patients aged $>60$ years [2]. All these factors contribute to the need for ICU admission in the elderly. Fuchs et al found that the rate of ICU admission was higher among the elderly population [3].

The impact of old age on ICU admission and outcome has been a subject of interest. Old age has been observed to be associated with poor outcome after ICU admission regarding mortality [4]. This has led to some clinicians refusing to admit older patients into the ICU reserving same for younger patients. To buttress this assertion, Sinuf and colleagues found that age and severity of illness were most strongly associated with a refusal to admit to the ICU [5]. Despite this, it is also known that age alone contributes little to patients' outcome in the ICU. Other factors that have been reported as more important determinants of outcome include severity of illness, admitting diagnosis and the type of interventions required such as mechanical ventilation and ionotropic support [6].

From the preceding, it becomes imperative to study the characteristics of critically ill elderly patients admitted into a level III ICU of a tertiary hospital in a Sub-Saharan African country. This will hopefully be useful in the care of this group of patients in terms of resource allocation and rationing a scarce resource like the ICU in this part of the world (Figure 1).

\section{Methodology}

The Intensive Care Unit of the University of Benin Teaching Hospital is a level III, multi-disciplinary seven-bed unit. Patients of all age groups are referred from different sub-specialties in the hospital and are co-managed with the referring specialists and consultants from the department of Anaesthesiology of the hospital.

This was a retrospective, case-control study. Patients aged 65 years and above admitted to the unit from January 2012 to June 2013 were included in the study. Hospital records including admission and discharge register of the ICU and nurses' report book were examined. A patient before and after each elderly patient in the register were recruited to serve as controls in the study. Data obtained included socio-demographic characteristics, diagnosis at admission and therapeutic interventions. Others included patients' length of stay in ICU and outcome (Figure 2).

Data obtained were entered into a proforma and analyzed with SPSS version 16. Continuous data were presented as frequencies and percentage. Parametric data were analyzed with chi-square and Fischer's exact test. A p value of $<0.05$ was considered statistical significance.

Correspondence to: Kingsley Ufuoma Tobi, Department of Anaesthesiology, University of Benin Teaching Hospital, Benin City, Nigeria, Tel: 08033600018; E-mail: tobikingsley@yahoo.com., tobikingsley265@gmail.com

Received: September 18, 2017; Accepted: October 28, 2018; Published: November 04, 2017 


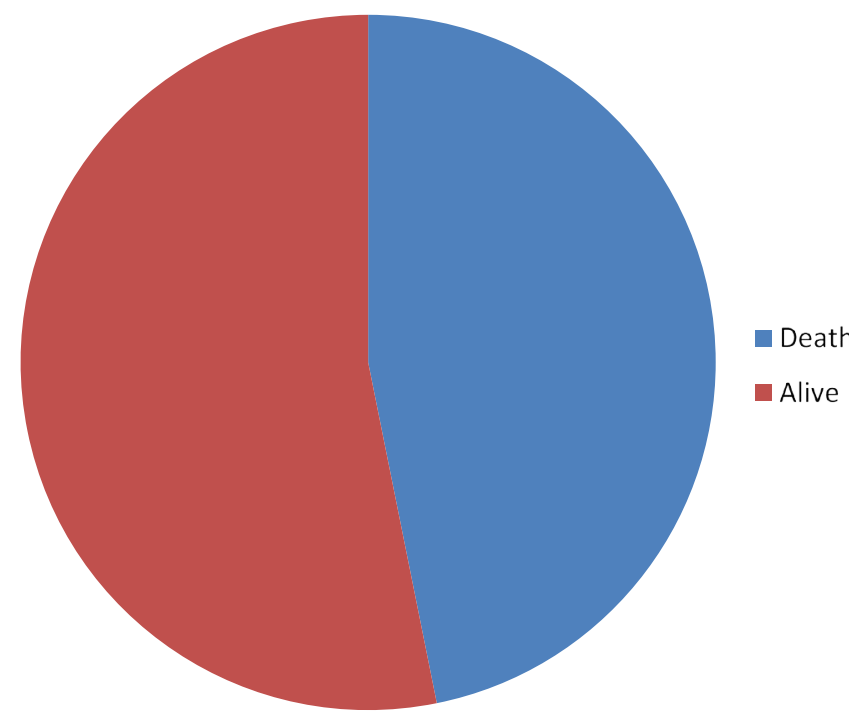

Figure 1. Elderly patients' outcome in the ICU. Mortality rate among elderly patients admitted in the $\mathrm{ICU}=46.8 \%$.

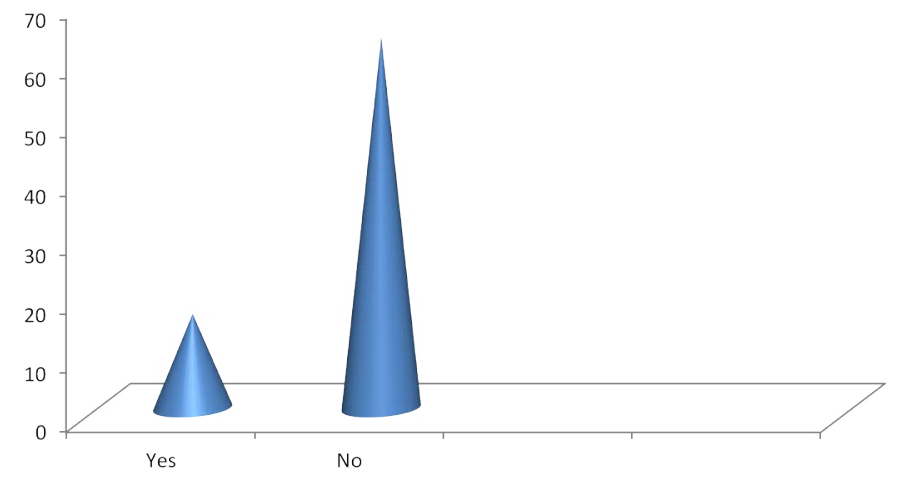

Figure 2. Mechanical ventilation among elderly patients.

\section{Results}

Out of 627 patients admitted to the ICU during the study period, seventy-nine (79) patients were aged 65 years and above constituting $12.6 \%$ of total ICU admission. Patients aged between 65-70 years represented the highest number of patients accounting for $45.6 \%$. This was followed by patients aged between $71-75$ years $(21.5 \%), 76-80$ years (12.6\%), 81-85 years and patients aged $>85$ years made up $13.9 \%$. More male $(67.1 \%)$ than female (32.9\%) elderly patients were admitted into the unit Table 1.

Postoperative care accounted for the highest indication for ICU admission (41.8\%) for elderly patients from this study. Cerebrovascular accident (stroke) constituted $12.6 \%$ of indications for ICU admission and sepsis and traumatic brain injury accounted for $8.9 \%$ respectively Table 2 .

Mortality among elderly patients admitted to the ICU was $49.6 \%$ and $46.8 \%$ among the control. Although more elderly patients died in the ICU, there was no statistical difference in mortality between elderly and other patients managed in the ICU $(\mathrm{p}=0.690)$ Table 3.

About $20.3 \%$ of elderly patients were mechanically ventilated while on admission in the unit which was lower than in the younger patients $(32.4 \%)$. Statistical analysis shows that younger patients were about twice more likely to be mechanically ventilated than elderly patients. $(\mathrm{p}=0.05, \mathrm{OR}=1.855)$ Table 4.
As shown in Table 5, about $21.5 \%$ and $29.5 \%$ of the elderly and other patients in the ICU were transfused with blood respectively. Although more patients aged less than 65years were transfused with blood, the difference did not achieve statistical significance. $\mathrm{P}=0.200$ Table 6 showed the length of stay for the groups of patients. Although the number of elderly patients who stayed longer than seven (7) days in the ICU was higher than in the younger patients group, $64.6 \%$ and $54 \%$ respectively, it was not statistically significant. $\mathrm{P}=0.128$.

\section{Discussion}

This study revealed that only a small proportion of patients (12.6\%) admitted to our ICU belonged to the elderly age group with patients aged between 65-70 years constituting the highest number (45.6\%). In a cohort study about a decade ago, Bagshaw et al [4] also reported an ICU admission rate of about $14 \%$ for patients 80 years and above. This could be due to the fact that intensive care including advanced

Table 1. Socio-demographic characteristics of elderly patients

\begin{tabular}{|c|c|c|}
\hline Age (years) & Frequency & Percentage \\
\hline $65-70$ & 36 & 45.6 \\
\hline $71-75$ & 17 & 21.5 \\
\hline $76-80$ & 10 & 12.6 \\
\hline $81-85$ & 5 & 6.3 \\
\hline$>85$ & 11 & 13.9 \\
\hline Total & 79 & 100 \\
\hline Sex & & \\
\hline Male & 53 & 67.1 \\
\hline Female & 26 & 32.9 \\
\hline Total & 79 & 100 \\
\hline & & \\
\hline
\end{tabular}

Table 2. Diagnosis

\begin{tabular}{|c|c|c|}
\hline Diagnosis & Frequency & Percentage \\
\hline Post-surgery & 33 & 41.8 \\
\hline Traumatic brain injury & 7 & 8.9 \\
\hline Cardiovascular accident & 10 & 12.6 \\
\hline Sepsis & 7 & 8.9 \\
\hline Cancers & 6 & 7.6 \\
\hline Intracranial haemorrhages & 5 & 6.3 \\
\hline Others & 11 & 13.4 \\
\hline Total & 79 & 100 \\
\hline
\end{tabular}

Table 3. Mortality between case (elderly patients) and control

\begin{tabular}{|c|c|c|}
\hline Case & Control & Total \\
\hline Death (37) & Death (69) & 106 \\
\hline Alive (42) & Alive (70) & 112 \\
\hline Total 79 & 139 & 218 \\
\hline $\mathrm{P}=0.690$ & & \\
\hline
\end{tabular}

Table 4. Ventilated patients between case and control

\begin{tabular}{|c|c|c|}
\hline Case & Control & Total \\
\hline Yes (16) & Yes $(45)$ & 61 \\
\hline No (63) & No $(94)$ & 157 \\
\hline Total 79 & 139 & 218 \\
\hline $\mathrm{P}=0.051$ OR $=1.855$ & & \\
\hline
\end{tabular}

Table 5. Blood transfusion between case and control

\begin{tabular}{|c|c|c|}
\hline Case & Control & Total \\
\hline Yes (17) & Yes $(41)$ & 58 \\
\hline No (62) & No (98) & 160 \\
\hline Total 79 & 139 & 218 \\
\hline $\mathrm{P}=0.200$ & & \\
\hline
\end{tabular}


Table 6. Length of stay in ICU between elderly patients and control

\begin{tabular}{|c|c|c|c|}
\hline LOS (days) & Elderly (case) & Control & Total \\
\hline$<1-7$ & $28(35.4 \%)$ & $64(46 \%)$ & 92 \\
\hline$>7$ & $51(64.6 \%)$ & $75(54 \%)$ & 126 \\
\hline Total & 79 & 139 & 218 \\
\hline $\mathrm{P}=0.128$ & & & \\
\hline
\end{tabular}

life support and therapies are often withheld for this age group [7]. In addition, the benefit of ICU care in terms of mortality and functional abilities following discharge remain unsatisfactory [4]. This may also contribute to the low admissions into the ICU for this age group.

Despite this low admission rate of the elderly into the ICU, it has been projected that the number will steadily increase. Blot and colleagues reported that the number of elderly patients aged 75 years and older rose by about 33\% between 1992-1996 and 2002-2006 [8]. This may be due to an increasing demand for ICU care due to aging of the population and increase in conditions like severe sepsis or highrisk surgery that will require care in the ICU [9]. It is therefore of urgent importance that appropriate admission criteria for this unique age group and protocol for their management in the ICU should be developed.

Fowler and co-workers in a study on sex and gender difference in the delivery and outcome of critical care observed that elderly men were more frequently admitted in the ICU than their female counterparts, despite same admission type and severity of illness [10]. In our study, we found that the number of elderly men admitted to the ICU was twice that of the women. It is difficult to adduce reasons for this observation. However in an African setting where the loss of a man is considered more devastating, it may be understandable why more men were admitted to the unit compared to women of this same age group.

Different reasons accounted for ICU admission of the elderly patients in this study. We found that postoperative care constituted the highest indication for ICU admission, followed by cerebrovascular accident (stroke), sepsis and traumatic brain injury. Surgical intervention in patients aged 80 years and above was the main indication for ICU admission in the study conducted by Bagshaw and co-workers [4]. The need for close monitoring following surgery is expectedly higher in the elderly than the younger patients. This may be due to the presence of comorbidities and delicate nature of surgeries in the elderly. The availability of invasive monitoring with alarm system and a higher nurse: patient ratio make the ICU ideal place for postoperative care of the elderly surgical patients.

We could not ascertain if ICU admission was planned before surgery for this group of patients or intraoperative events necessitated the admission due to the retrospective nature of our study. Ejiro and Edomwonyi [11] had studied admission into ICU from the operative room at the same center from 2007-2009. They found that $3.4 \%$ of all surgical cases performed under general anaesthesia were admitted into the ICU. Out of these, $42.8 \%$ were planned admissions and they identified higher American Society of Anesthsiologists (ASA) physical status, emergency procedures, major surgeries and prolonged duration of surgery as risk factors for ICU admission. However, the authors did not make any comments on age as a possible risk factor for planned admission to ICU postoperatively.

Although, old age has been associated with poor outcome after ICU admission [4], our study showed that there was no significant difference between mortality between the elderly and younger patient population. Therefore, factors other than age itself may play a more direct influence on ICU outcome. Previously, indications for ICU admission, severity of illness and interventions have been documented as determinants of outcome in our centre [6].

Mechanical ventilation was commoner among the younger age group than elderly patients as less than one quarter were ventilated during their ICU stay. Some reasons may be advanced for this finding. It has been noted that ventilatory support is often cautiously withheld in the elderly age group which may account for the low rate of ventilatory support we observed [7]. Many of the elderly patients in this study did not require ventilatory assistance as the commonest indication for their admission was close monitoring following surgery. In addition, this may be due to the associated difficulty in weaning off the support due to presence of comorbidities such as chronic obstructive airway diseases (COAD) which is common in this age group.

Patients' length of stay in the ICU had been reported to be significantly related to admitting units/indication for ICU admission [6] In that study, patients who had neurosurgical procedures stayed longer than other groups of patients in the ICU. This finding did not however take into consideration age differences of the patients studied. In this present study, although more patients in the elderly age group stayed longer than seven days compared to other patients, it was not statistically significant. As a result, it could be opined that age is not an independent factor for length of ICU stay.

\section{Conclusion}

Although the number of elderly patients admitted into our ICU was small, with a high mortality, it is expected to increase with improved medical care and aging of the population. Appropriate admission criteria for this unique age group and protocol for their management in the ICU should be developed to improve outcome.

\section{Limitations}

The retrospective nature of this study makes some limitations inevitable. The results of investigations done for these patients could not be retrieved for analysis. Besides, we could not ascertain if there was any advanced directive or "Do Not Resuscitate" order for any of our patients; although this is uncommon in our environment. The absence of severity of illness scoring like APACHE score also added to the limitations. Despite these obvious limitations, we have shown that although elderly patients constitute a small percentage of admission into the ICU, they have high mortality.

\section{References}

1. Martin GS, Mannino DM, Moss M (2006) The effect of age on the development and outcome of adult sepsis. Crit Care Med 34: 15-21. [Corssref]

2. Ewig S, Birkner N, Strauss R, Schaefer E, Pauletzki J, Bischoff H et al. (2009) New perspectives on community-acquired pneumonia in 388, 406 patients. Results from a nationwide mandatory performance measurement program in healthcare quality. Thorax 64: 1062-1069. [Corssref]

3. Fuchs L, Chronaki C E, Park S, Novack V, Baumfeld B, Daniel Scott et al. (2012) ICU admission characteristics and mortality rates among elderly and very elderly patients. Intensive Care Medicine 38: 1654-1661. [Corssref]

4. Bagshaw SM, Webb SA, Delaney A, George C, Pilcher D, Hart GK et al. (2009) Very old patients admitted to intensive care in Australia and New Zealand: a multi-centre cohort analysis. Crit Care 13: R45). [Corssref]

5. Sinuff T, Kahnamoui K, Cook DJ, Luce JM, Levy MM (2004) Rationing critical care beds: a systematic review. Crit Care Med 32: 1588-1597. [Corssref]

6. Tobi K U, Efobi CA (2015) Characteristics and outcome of critically ill patients admitted into a general intensive care unit in Nigeria. African Journal of Medical Health Sciences. 
Tobi KU (2017) Characteristics of critically ill elderly patients admitted to a tertiary intensive care unit in Nigeria and outcome of management

7. Hamel MB, Teno JM, Goldman L, Lynn J, Davis RB, Galanos AN et al. (1999) Patient age and decisions to withhold life-sustaining treatments from seriously ill, hospitalized adults. SUPPORT Investigators. Study to Understand Prognoses and Preferences for Outcomes and Risks of Treatment. Ann Intern Med 130: 116-125. [Corssref]

8. Blot S, Cankurtaran M, Petrovic M, Vandijck D, Lizy C, Decruyenaere J et al. (2009) Epidemiology and outcome of nosocomial bloodstream infection in elderly critically ill patients: a comparison between middle-aged, old, and very old patients. Crit Care Med 37: 1634-1641. [Corssref]
9. Angus DC, Wax RS (2001) Epidemiology of sepsis: an update. Crit Care Med 29: S109-S116. [Corssref]

10. Fowler RA, Sabur N, Li P, Juurlink DN, Pinto R, Hladunewich MA, Adhikari NK, et al. (2007) Sex- and age-based differences in the delivery and outcomes of critical care. CMAJ 177: 1513-1519. [Corssref]

11. Ejiro BA, Edomwonyi NP (2012) Audit of Intensive Care Unit admissions from the operative room: experience at the University of Benin Teaching hospital, Benin City, Nigeria. Journal of Medicine and Biomedical Research 11: 17-19.

Copyright: $@ 2017$ Tobi KU. This is an open-access article distributed under the terms of the Creative Commons Attribution License, which permits unrestricted use, distribution, and reproduction in any medium, provided the original author and source are credited. 